\title{
Improving the Operational Bandwidth of a 1-3 Piezoelectric Composite Transducer using Sierpinski Gasket Fractal Geometry
}

\author{
H. Fang, Z. Qiu, R. L. O’Leary and A. Gachagan \\ Centre for Ultrasonic Engineering \\ University of Strathclyde \\ Glasgow, UK G1 1XW \\ Email: haoyu.fang@strath.ac.uk
}

\author{
A. J. Mulholland \\ Department of Mathematics \\ University of Strathclyde \\ Glasgow, UK G1 1XH
}

\begin{abstract}
Wider operational bandwidth is an important requirement of an ultrasound transducer across many applications. It has been reported mathematically that by having elements with varying length scales in the piezoelectric transducer design, the device may possess a wider operational bandwidth or a higher sensitivity compared to a conventional device. In this paper, the potential for extending the operational bandwidth of a 1-3 piezoelectric composite transducer configured in a fractal geometry, known as the Sierpinski Gasket (SG), will be investigated using finite element analysis package PZFlex (Thornton Tomasetti). Two equivalent piezocomposite designs will be simulated: a conventional 1-3 piezocomposite structure and the novel SG fractal geometry arrangement. The transmit voltage response and open circuit voltage extracted from the simulations are used to illustrate the improved bandwidth predicted from the fractal composite design.
\end{abstract}

Keywords-fractal; Sierpinski Gasket; piezocomposite;

\section{INTRODUCTION}

The concept of a 'piezoelectric composite' ultrasound transducer is a well-established design [1-3]; it refers to an ultrasound transducer containing a piezoelectric material incorporating both an active piezoelectric phase and a passive polymer phase. Such piezoelectric composite configurations, when designed correctly, can attain high sensitivity whilst being well matched to low acoustic impedance load [4]. Piezoelectric composite ultrasound transducers with a wide operational bandwidth are preferred in many applications. For example, in under water sonar, a broadband transducer can provide better spatial resolution [5]. In NDT applications, a broadband transducer can be used for difficult material testing or replace several narrowband transducers each operating at a different frequency ranges [6]. Also, in biomedical applications, imaging performance can be enhanced by using a broadband transducer [7].

In general, there are three main methods to enhance the operational bandwidth of a composite transducer:

a) Improve the piezoelectric material properties

b) Modifying the matching/backing design

c) Optimising the composite structure, including the filler material properties
For enhancing the piezoelectric material properties, Yamada et al proposed a method of designing a broadband ultrasound transducer by creating a graded piezoelectric constant $\left(\mathrm{e}_{33}\right)$ in the thickness direction of a piezoelectric material [8]. For increasing the transducer bandwidth with an optimised matching design, Hossack and Auld reported a novel transducer design with an active piezoelectric matching layer [9]. Moreover, by optimising the structure of the composite design, the transducer bandwidth can also be improved. Harvey et al designed a random composite transducer with piezoelectric fibres to improve the device bandwidth [10]

In naturally occurring auditory systems, it is common to observe hearing organs comprising a number of different length scales. Such hearing organs exhibit extended operating bandwidth, examples include insects such as the bushcricket [11-13]. Importantly, the concept of engineered transducers comprising multiple length scales has been developed mathematically to evaluate potential gains in bandwidth by introducing such a concept [14-16]. These analytical models indicate that by having elements with varying length scales in the piezoelectric transducer design, the device may possess a wider operational bandwidth or a higher sensitivity compared to a conventional device. In this paper, a self-similar fractal geometry known as the Sierpinski Gasket (SG), shown in the Fig. 1, will be employed in order to improve the bandwidth of the 1-3 composite configuration transducer. This SG fractal geometry will be adapted as the structure of a piezocomposite design and the performance of this SG fractal composite will be compared with an equivalent conventional composite design.

The finite element (FE) analysis package, PZFlex (Thornton Tomasetti), will be used to simulate the transmission and reception response of each of these composite configurations to provide quantitative comparison between the SG fractal composite and the conventional composite designs. The FE simulation results show that an encouraging bandwidth improvement can be achieved by implementing the SG fractal geometry compared to the conventional composite design with a regular periodic structure. 


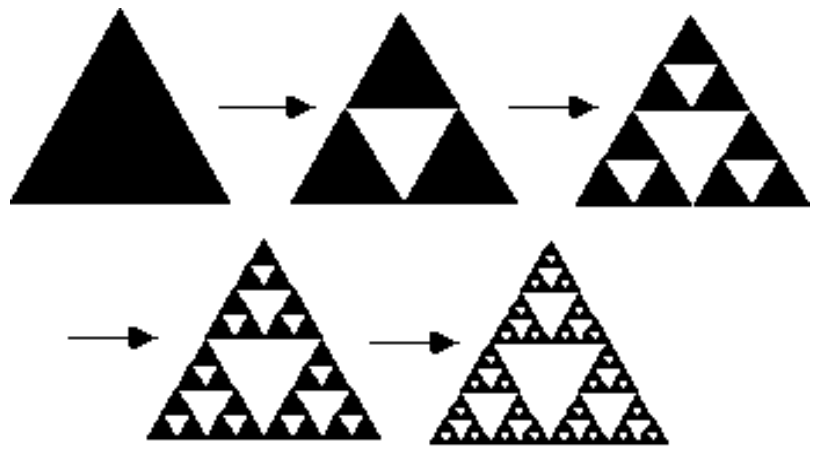

Fig. 1: The First Four Generations of the Sierpinski Gasket Geometry

\section{MethodOLOGY}

In order to explore the problem space, two 3D FE models are developed. Fig. 2 illustrates the generation level IV SG fractal composite design, where the active phase of this SG composite is comprised of triangular ceramic pillars with different lateral length scales and Fig. 3 is the equivalent conventional parallelepiped 1-3 composite design. Consistency is maintained between the two composite designs by ensuring each device has the same fundamental design parameters: the PZT5H ceramic and CY1300/HY1301 polymer are chosen to be the active phase and passive phase material respectively; the ceramic volume fraction and thickness of two composite designs are both $68.4 \%$ and $2.6 \mathrm{~mm}$ respectively; each device comprises the same matching layer arrangement. Furthermore, the aspect ratio of the fourth generation triangular pillars in the SG fractal composite is the same as the conventional composite design.

The FE models simulate the operation of both devices when matched to a water load, via a dual matching layer scheme [1718]. Details of the matching layer configuration are provided in Table I, where Layer I is the outer layer closed to the water load and Layer II is the inner layer closed to the front face of the composite.

Table I: Dual Matching Layer Specification

\begin{tabular}{|c|c|c|}
\hline & Layer I & Layer II \\
\hline Material & CY221/HY956EN & $\begin{array}{c}\text { Alumina filled (70\% } \\
\text { w/w) } \\
\text { CY1301/HY1300 }\end{array}$ \\
\hline Thickness $(\boldsymbol{\mu}$ m) & 580 & 1093 \\
\hline $\begin{array}{c}\text { Acoustic Impedance } \\
\text { (MRayl) }\end{array}$ & 2.68 & 6.96 \\
\hline
\end{tabular}

The performance of these two devices are assessed by considering the transmit voltage response (TVR) and the open circuit receiving voltage response (OCV) extracted from the simulations.

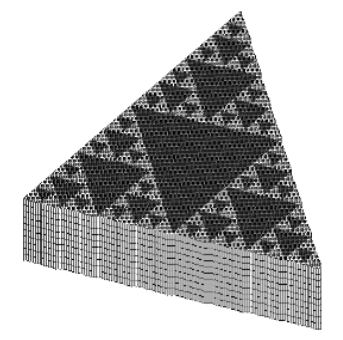

Fig. 2: SG Fractal Composite FE Model.

(Black: Ceramic Pillar; Gray: Polymer Filler)

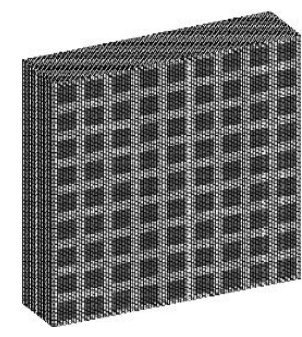

Fig. 3: Conventional Composite FE Model.

(Black: Ceramic Pillar; Gray: Polymer Filler)

\section{RESULT}

The electrical impedance magnitude spectra of the SG composite and conventional composite are simulated operating in a water load and shown in Fig. 4. It can be seen that the conventional composite device has a single mode around $575 \mathrm{kHz}$, with the SG device exhibiting a multi-modal characteristic and an impedance minimum at $580 \mathrm{kHz}$, which is close to the electrical resonance of the conventional device.

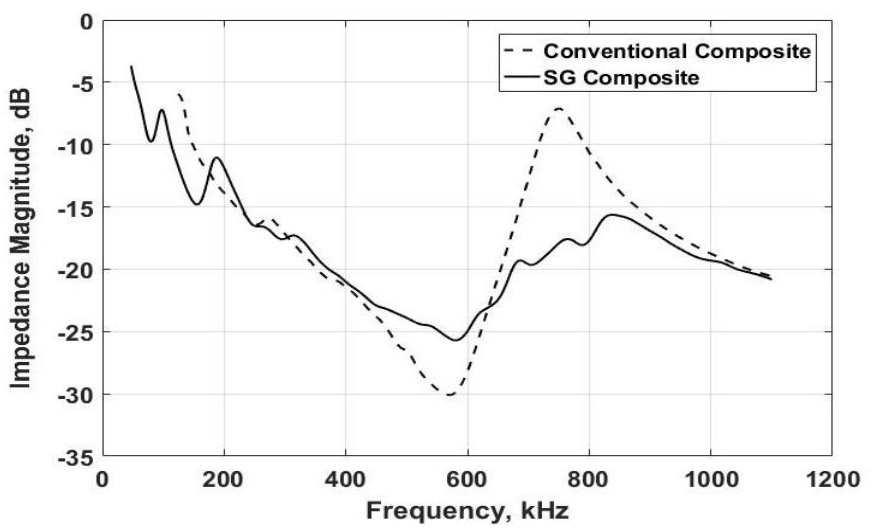

Fig. 4: Impedance Magnitude Spectrum of the SG and the Conventional Composite

Applying the same matching layer arrangement to both the SG and the conventional transducers, the predicted TVR and OCV are shown in Fig. 5 and Fig. 6, respectively.

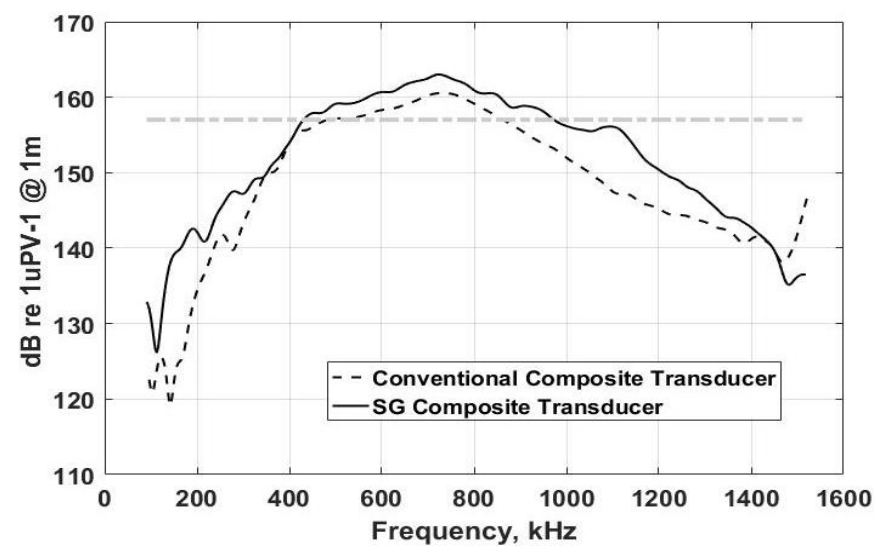

Fig. 5: TVR Spectrum of the SG and the Conventional Composite Transducer 


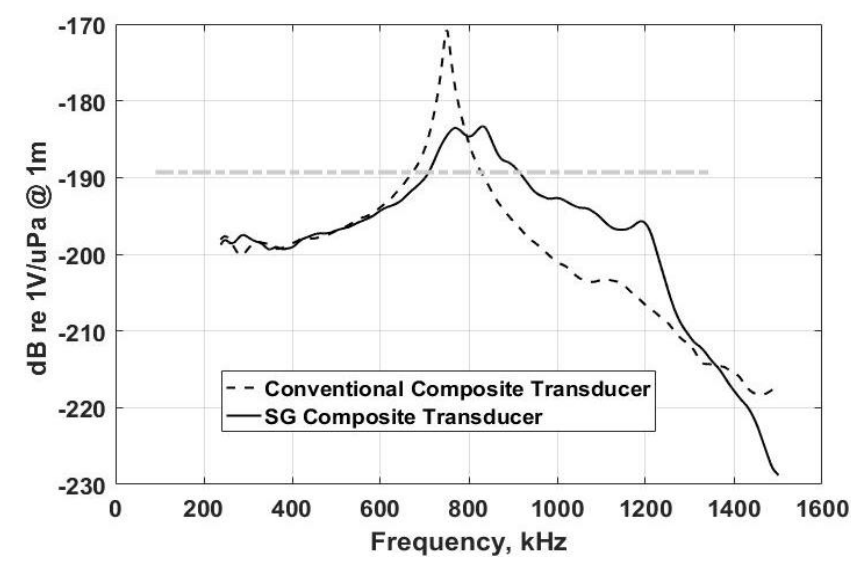

Fig. 6: OCV Spectrum of the SG and the Conventional Composite Transducer

For comparison between the two transducers, a common noise floor (shown as the grey dashed line in the TVR and OCV spectra) has been defined for both the $S G$ and the conventional device, which is $6 \mathrm{~dB}$ below the peak gain of the SG composite in transmission and reception mode. The operational bandwidth results for both devices are shown in Table II.

Table II: Transmission and Reception Bandwidth Improvement with respect to Conventional Composite Device

\begin{tabular}{|c|c|c|}
\hline & $\begin{array}{c}\text { Transmission } \\
\text { Bandwidth }\end{array}$ & $\begin{array}{c}\text { Reception } \\
\text { Bandwidth }\end{array}$ \\
\hline $\begin{array}{c}\text { Conventional } \\
\text { Composite }\end{array}$ & $52.3 \%$ & $21.7 \%$ \\
\hline SG Composite & $75.5 \%$ & $27.7 \%$ \\
\hline Improvement & $44.5 \%$ & $27.6 \%$ \\
\hline
\end{tabular}

It can be seen from the Table I that by using the SG fractal geometry as the structure of a piezoelectric composite design, the operational bandwidth of the device can be extended in both transmission and reception mode. In the transmission mode, the operational bandwidth has been improved by $44.5 \%$ with respect to the conventional composite design and in the reception mode, a bandwidth enhancement of $27.6 \%$ has been achieved with respect to the conventional design.

\section{DISCUSSION}

In order to further explore the influence of using the SG fractal geometry as the structure of a piezocomposite design, the TVR spectrum of the SG composites from Level I to Level VI are compared to equivalent conventional composite designs - for each case the data are shown in Fig. 7. It can be seen that the SG composite at the first three levels behaved approximately the same as the conventional composite. However, as the generation level of the SG composite increases beyond three, the SG composite starts to show a better performance compared to the conventional composite design. Considering the difficulties of the manufacturing process, a

Level IV SG fractal composite is considered a good initial choice for manufacture.
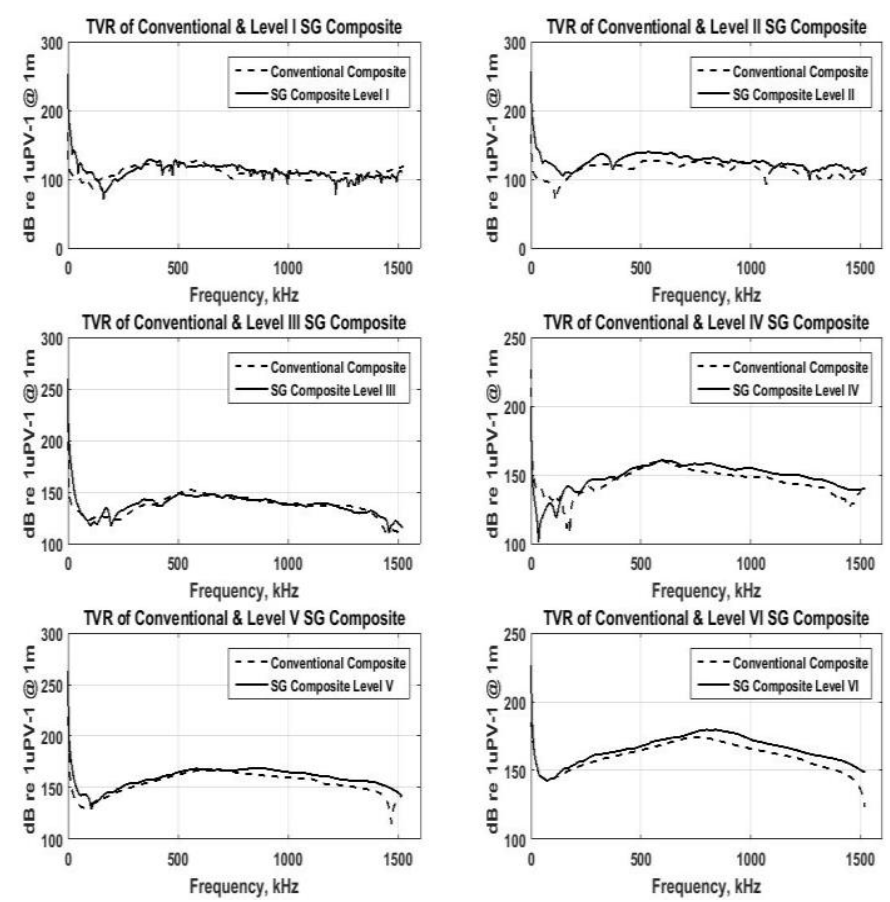

Fig. 7: TVR of the Conventional and the SG Composite (Level I to Level VI)

In addition to using the SG fractal geometry as the structure of the composite design, the influence of using a SG fractal electrode pattern on a conventional triangular-cut 1-3 composite has also been investigated. A 3D FE model of a 1-3 triangular cut composite substrate is shown in Fig. 8. This 1-3 triangular-cut composite substrate was first electroded using a fractal mask (shown in Fig. 9) and then the active area uniformly electroded (shown in Fig. 10).

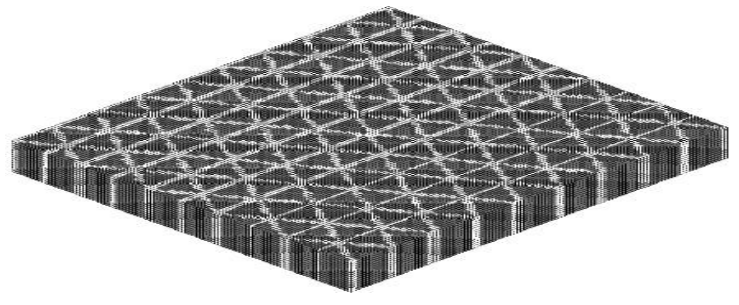

Fig. 8: 1-3 Triangular Cut Composite Substrate (Black: Ceramic Pillar; Gray: Polymer Filler)

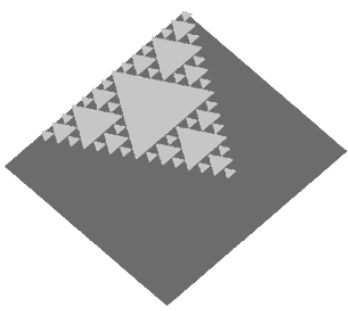

Fig. 9: Fractal Electrode Configuration

(Gray: Top Electrode; Black: Ground

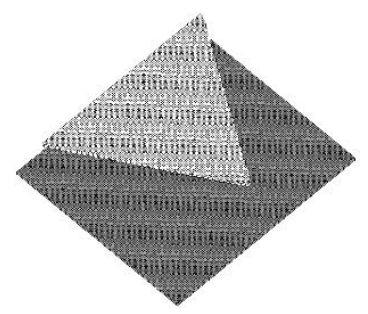

Fig. 10: Full Electrode Configuration

(Gray: Top Electrode; Black: Ground) 
However, the simulation results in Fig. 11 and Fig. 12 show that by using a fractal electrode pattern rather than a full electrode, the operational bandwidth of the composite would not be enhanced in neither transmission nor reception mode.

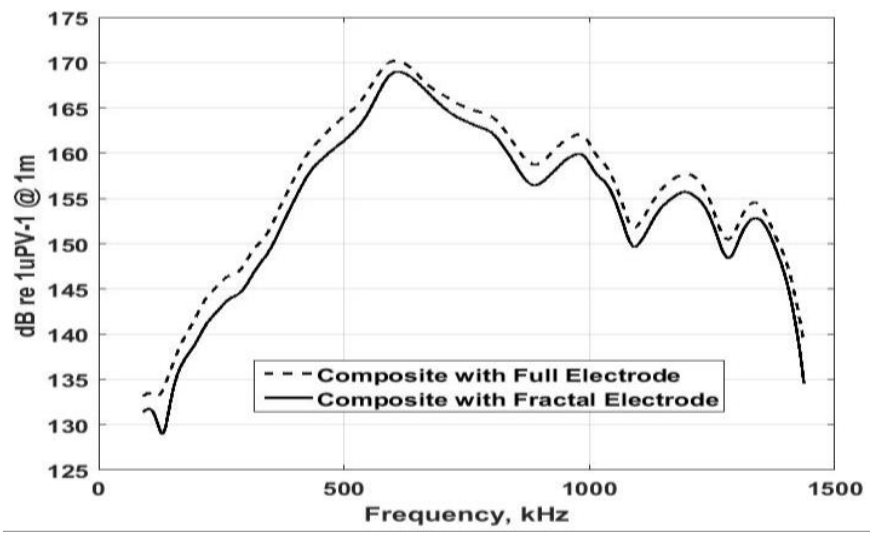

Fig. 11: TVR Spectrum of the Full and the Fractal Electroded Composite

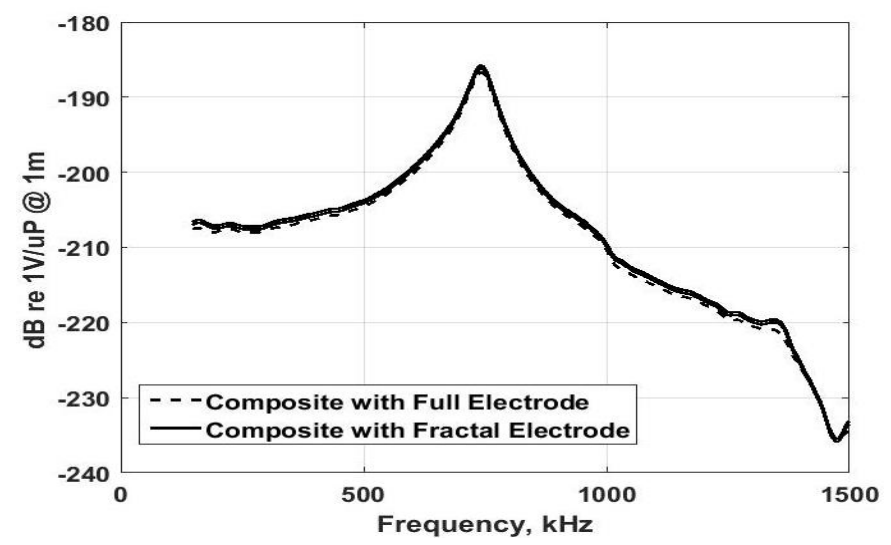

Fig. 12: OCV Spectrum of the Full and the Fractal Electroded Composite

\section{CONCLUSION.}

This paper describes the implementation of the SG fractal geometry as the structure of the piezocomposite design in order to improve the bandwidth of piezocomposite transducers. The FEA simulation results show that when applying the SG fractal geometry at generation levels greater than level III, a wider bandwidth can be achieved in both transmission and reception mode compared to an equivalent conventional design. However, the performance of a conventional composite device cannot be improved by replacing the full electrode with a fractal electrode. As the result, the manufacture of an initial prototype Level IV SG fractal composite transducer is currently being undertaken based on the positive simulation results and the authors look forward to sharing experimental results in the future.

\section{ACKNOWLEDGMENT}

The core funding for Mr Fang's studentship has been provided by the University of Strathclyde and the work has been carried out under the auspices of the UK Research Centre for NDE (EPSRC Grant EP/L022125/1).

\section{REFERENCES}

[1] R. E. Newnham, D. P. Skinner, and L. E. Cross, "Connectivity and piezoelectric-pyroelectric composites," Materials Research Bulletin, vol. 13, no. 5. pp. 525-536, 1978 .

[2] T. R. Gururaja, R. E. Newnham, K. A. Klicker, S. Y. Lynn, W. A. Schulze, T. R. Shrout, and L. J. Bowen, "Composite Piezoelectric Transducers," in IEEE Ultrasonics Symposium, vol. 53, no. 9, pp. 576581. 1980

[3] G. Hayward and J. Bennett, "Assessing the influence of pillar aspect ratio on the behavior of 1-3 connectivity composite transducers," IEEE Transactions on Ultrasonics, Ferroelectrics, and Frequency Control, vol. 43, no. 1, pp. 98-108, 1996.

[4] J. T. Bennett, "Design of 1-3 piezocomposite hydrophones using finite element analysis," IEEE Transactions on Ultrasonics, Ferroelectrics, and Frequency Control, vol. 44, no. 3, pp. 565-574, 1997.

[5] S. Cochran, M. Parker, and P. Marin-Franch, "Ultrabroadband single crystal composite transducers for underwater ultrasound," in IEEE Ultrasonics Symposium, vol. 1, no. c, pp. 231-234. 2005,

[6] P. Marin-Franch, I. Pettigrew, M. Parker, K. J. Kirk, and S. Cochran, "Piezocrystal-polymer composites: New materials for transducers for ultrasonic NDT," Insight Non-Destructive Test. Cond. Monit., vol. 46, no. 11, pp. 653-657, 2004.

[7] E. S. Ebbini, C. Simon, H. Lee, and Wonjoon Choi, "Self-guided ultrasound phased arrays for noninvasive surgery," in IEEE Ultrasonics Symposium. Proceedings. International Symposium, vol. 2, pp. 1427 1430,1999

[8] K. Yamada, D. Yamazaki, and K. Nakamura, "Broadband ultrasound transducers using a plate with a graded piezoelectric constant formed by an internal temperature gradient," in IEEE Ultrasonics Symposium., pp. 1017-1020, 2000

[9] B. A. Hossack, J. A., Auld, "Multiple layer transducers for broadband applications," in IEEE Ultrasonics Symposium, pp. 605-610, 1991.

[10] G. Harvey, A. Gachagan, J. W. MacKersie, T. McCunnie, and R. Banks, "Flexible Ultrasonic Transducers Incorporating Piezoelectric Fibres," IEEE Transactions on Ultrasonics, Ferroelectrics, and Frequency Control, vol. 56, no. 9, pp. 1999-2009, 2009.

[11] W. Rössler, "Postembryonic development of the complex tibial organ in the foreleg of the bushcricket Ephippiger ephippiger (Orthoptera, Tettigoniidae)," Cell Tissue Res, vol. 269, pp. 505-514, 1992.

[12] W. Rössler, "Functional morphology and development of tibial organs in the legs I, II and III of the bushcricket Ephippiger ephippiger (Insecta, Ensifera)," Zoomorphology, vol. 112, no. 3, pp. 181-188, 1992.

[13] W. Rössler, A. Hübschen, J. Schul, and K. Kalmring, "Functional morphology of bushcricket ears: comparison between two species belonging to the Phaneropterinae and Decticinae (Insecta, Ensifera)," Zoomorphology, vol. 114, no. 1, pp. 39-46, 1994.

[14] E. A. Algehyne and A. J. Mulholland, "A finite element approach to modelling fractal ultrasonic transducers," IMA J. Appl. Math., 80(6), 1684-1702, 2015.

[15] L. Orr, A. J. Mulholland, R. L. O’Leary, and G. Hayward, "Analysis of ultrasonic transducers with fractal architecture," Fractals, vol. 16, no. 4, pp. 333-349, 2008.

[16] A. J. Mulholland and A. J. Walker, "Piezoelectric Ultrasonic Transducers With Fractal Geometry," Fractals, vol. 19, no. 04, pp. 469-479, 2011.

[17] D. Callens, C. Bruneel, and J. Assaad, "Matching ultrasonic transducer using two matching layers where one of them is glue," NDT E Int, vol. 37, no. 8, pp. 591-596, 2004.

[18] R. Hill, S. M. A. Ei-dardiry, and R. Gordon, "A theory for optimization in the use of acoustic emission transducers," vol. 67, no. 2, pp. 673-682, 1980 . 